LA-UR -83-789

-

I.R-U1:- $-83-789$

DE: $0100 \Omega ?$

TITLE NONLINEAR hY'IRODYNAMICS, Lecture 9

AUTHOR,SI

A

;

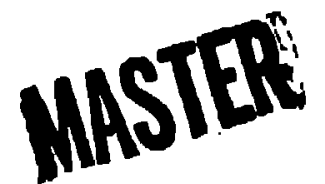

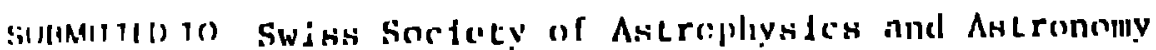

Silits Pre, Swltzorland, Mirch 2.l-26, 1983

\title{
IMC'A.AIMER
}

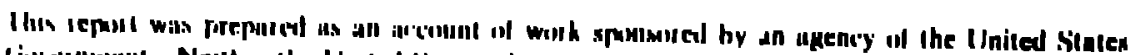

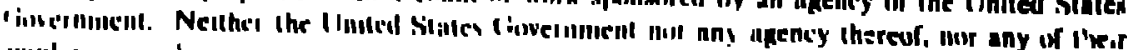

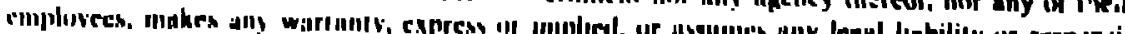

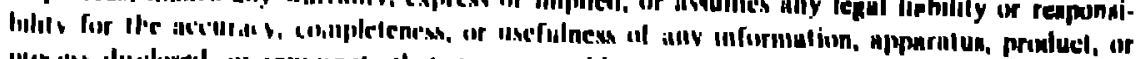

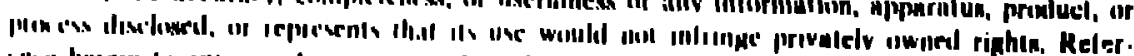

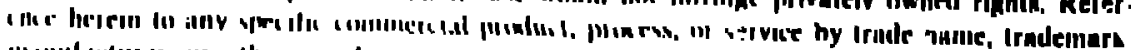

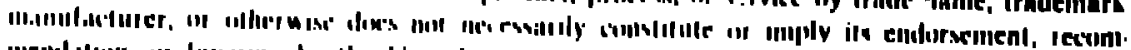

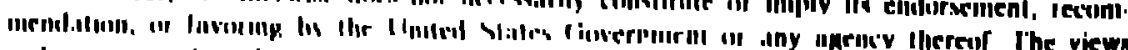

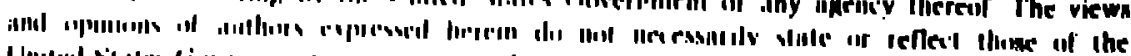

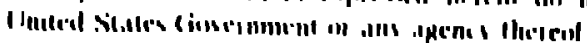

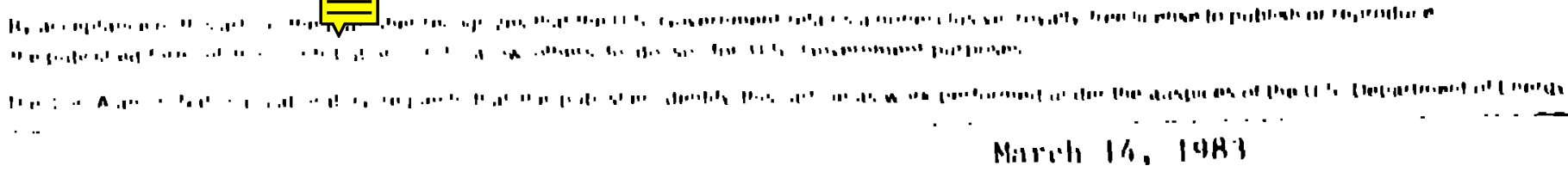

Mirill 1/, IOHS 


\section{NONLINEAR HYDRODYNAMICS}

We now come to a very sophisticated method for calculating the stability and pulsations of stars which make contact with actual observations of the stellar behavior. Hydrodynamic calculaticns are very simple in principle. Conservation of mass can be accounted for by having mass shells that are fixed with their mass for all time. Motions of these shells can be calculated by taking the difference between the external orce of gravity and that from the local pressure gradient. The conservation of energy can be coupled to :his momentum conservation equation to give the current temperatures, densities, pressures, and apacicies at the shell centers, as well as the positions, velocities, and accelerations of the mass shell interfaces. Energy flow across these interfaces can be calculated from the current conditions, and this energy is partitioned between internal energy and the work donc on or by the mass shell. We will discuss here only the purely radial case for hydrodynamics because it is very useful for stellar pulsation studies.

The onc-dimensional initial value computer programs for calculiting stellar pulsations are fow in number. Christy (1964) published the first description of a stellar hydrodynamics cole. It was based on Lechnigues developed at los Alamos in earlier days. We also used the same gencral methods and published a description in a piper by cox, Brownlec, and bilers (1966). There have beon severaj programs since, incl Lheir descriptions are given by stobie (1969), Bakre and von Senglusch (1969), Hende dud Davili (1971), Stollingwerf (1974), Wood (1974), Spingengerg (197!), Kilp) (1975), Castor, Dilvis, and Davisoll (1977), and Vemury and stothers (1978).

All these pograms were writlen lor stuelies of cipheids and RK

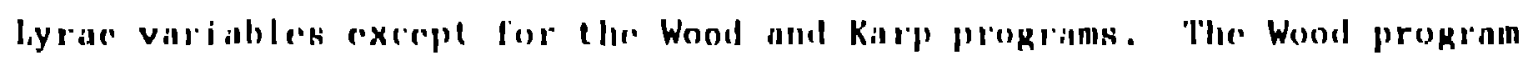
has berll lesed ouly lof calculations of Mira variables, and the Karp

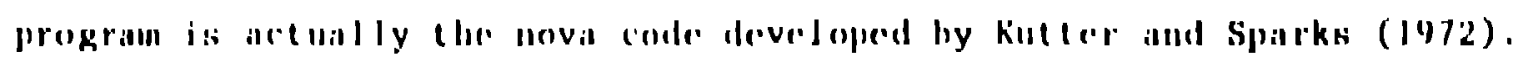
Mira pulsidions hove also been studied with the christy code (Keeley

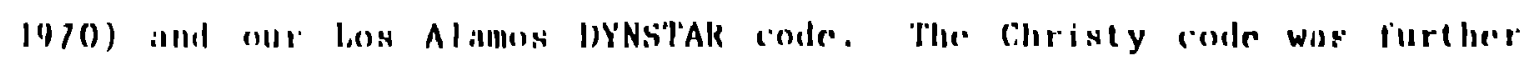

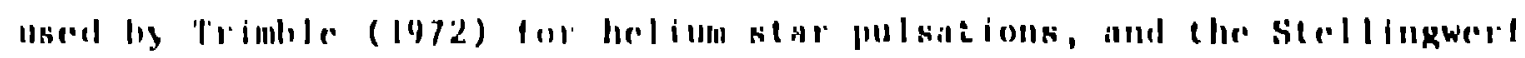

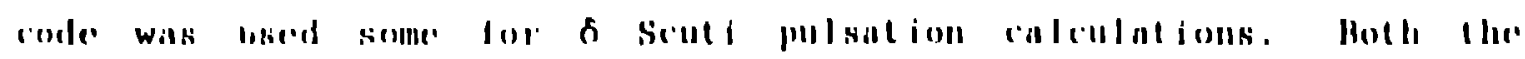

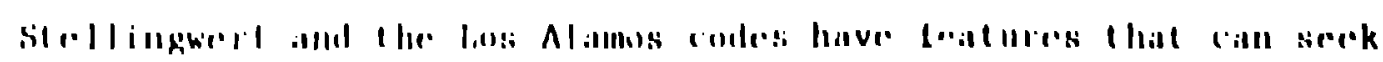


strictly periodic solutions by a relaxation technique, but this valuable procedure for Cepheids will not be discussed here because it does not work for the very slowly decaying pulsations of the upper main sequence stars.

Appenzeller (1970), Ziebarth (1970), and Talbot (1971) have written computer codes for the calculation of pulsations of very massive upper main sequence stars. Later Papaloizou (1973), using essentially the Christy techniques, has calculated the hydrodynamics of stars masses between 70 and 210 solar masses. These calculations were not very detailed and were not very definitive in their predictions of possible pulsationally induced mass loss. Nowadays the facts seem to be that stellar winds produce so much mass loss that even the lowest mass considered by these authors, sixty solar masses, have very short lives. It is still possible, however, that pilsation is responsible for the very high mass loss rates seen in stars such as the Wolf-Rayet stars.

The Appenzeller, Kutter-Sparks, Gtellingwerf, Castor, and our DYNSTAR codes are complelely implicit. That means that the equalions are time centered at least halfway across the time step, and therefore the solution nocessaljly juvolves ilerations for quallities al the advanced time. The Courant time step limit (see Richlmyer and Murton $1967)$ is not necessary for Lhese codes, and if Lhe accuracy allows a lirger lime step, it can be used. The encrgy equalion is solved implicilly, however, in all the above described mumerical programs.

The current version of the DYNSTAk progran will he described here. As ond might expect, all these abovermentioned proglians have cuolverl or died, and the published deseriptions are generally not accurate. Iho' current DYNSTAR flogram, male completely impliceit in 19is is alctually a

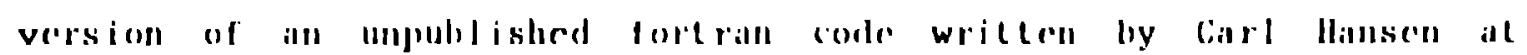
Boulder, hased on our published concepls, and some of his, ind rinally shippod biak to losi Alamos!

Jhe starting conliguration ol the lagrangian miss shells is that

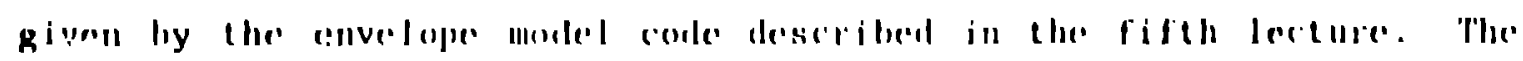

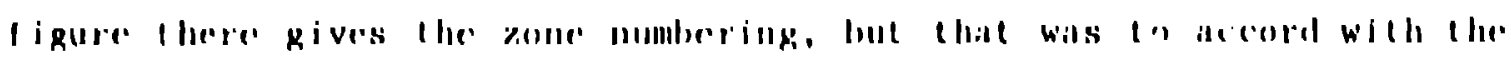

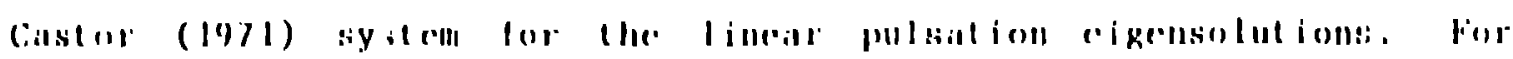

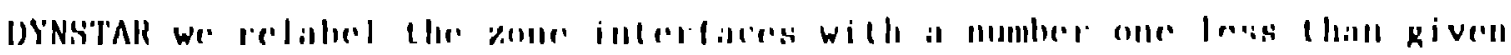

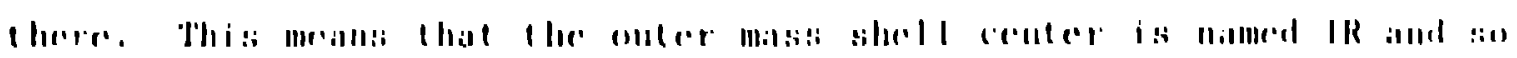

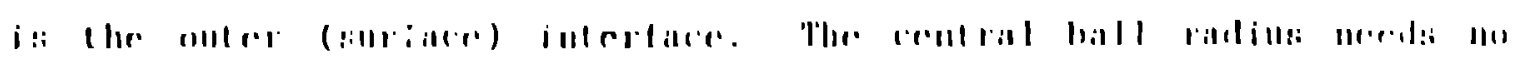


number because it remains fixed in space for a11 time and produces a fixed luminosity.

Figure 1 gives the two basic equations for DYNSTAR. These are the momentum and the energy equations. The equation for mass conservation in our Lagrangian system is only an auxiliary one which gives the - specific volume of each mass zone at the beginning of each tinestep and during the implicit method iterations for the end of the timester. The hydrodynamic solution is really just a series of models spaced a timestep apart, where this imestep is as large as possible to retain accuracy of the solution.

Values of all the variables are known at time $n$. For the needed values at the next time $n+1$, there is an axtrapolation of the quantities and then a set of a few iterations to improve these values so that the equations are accurately satisfied. Because we have what is called a completely implicit method, there is no mathenatical (Courant) stability limitaiion on the timestep as there is when the hydrodynamic behavior is calculated explicitly.

1-D) I.AGRANG]AN HYDROIOYNAMICS-TWO VARIABLES MASS, MOMENTUM, AND FNEKGY CONSERVATJON

$$
\begin{aligned}
& \Delta t=t^{11+1}-L^{n} \text {; inlerface } I \text {; mass zone } I ; l \leq I \leq I R \\
& V=\frac{4 \pi}{3 M_{1}} \cdot\left(r_{1}^{3}-r_{I-1}^{3}\right) \\
& \mu=\ddot{r}_{1}^{\prime \prime+l}+2 \dot{r}_{1}^{n} / \Delta t-2 \Delta r_{1}^{n+l_{2}} /(\Delta t)^{2}=0 \\
& \mathrm{I}:=\Delta r_{1}^{n+\frac{1}{2}}-\Delta r_{1}^{n+\frac{1}{2}}-\mu_{1}^{n+\frac{l}{2}} \Delta v_{1}^{n+!}=0
\end{aligned}
$$

ligure 1. I'le baste equat ions of mass, momenlum, and energy conservilt ion are integlited over limesteps fnr ali the liagrangiall miss molle's.

OII so-called momentum expuation is really all expression relatiug

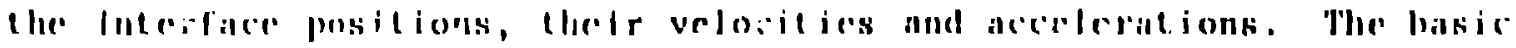
guintity as the arecelerntion which is time centered by nupraging the

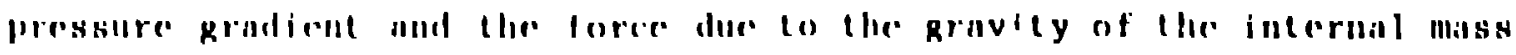

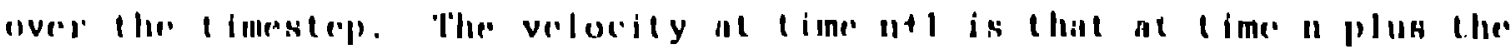

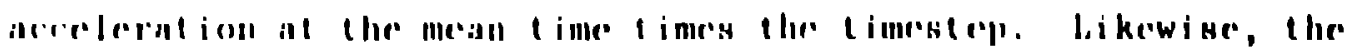


difference in the interface radius between time $n+1$ and time $n$, that is, $\delta r$ centered at time $n+\frac{1}{2}$, is the velocity centered at time $n+\frac{1}{2}$ times the timestep. This velocity at the midtime is taken as the mean of its value at time $n$ and $n+1$. Combining all these things results in what we call the momentum equation, and $j t$ is iterated together with all the

provisional quantities such as temperature, specific volume, pressure, etc at time $n+1$ until the equation equals zero.

Our energy equation is the first law of thermodynamics. Energy flowing into or out of the mass shell by radiation, conduction, or convection plus the energy introduced into the mass shell by nuc]ear burning is partitioned between internal energy and work according to this equation. Time centering of all these terms means that the variables must bo guessed and iteratively imprcved for lime $n+1$, jusl as for the momentian equation. Convergence for both of the basic equations is reached wiell the equalions attain values small comparcd to lheir largest terms.

The momentum equation that describes the zone interface acrelerition is giverl in Fig!re 2. Fraley (1968) has shown that to conscrve encrgy exictly through the fontire cuvelope model, it is neassitry to define the inein sfuare of the interface radius in the way given which involves holh limes 11 ind n+1. Also it is mecessiry lo lase lad arcelelation due le gravity at a mean radiass as given again involving the radius at both emb of the timescep. 'lhe figure also silow's seme detail aboul the monaliabatid term ol the rnergy cquation. laninosity hy ralialion and conduction is cislculated with the normal Rossclind diffusion equation where the $k$ includes both adialive and (o) moluct ive ('out ributions.

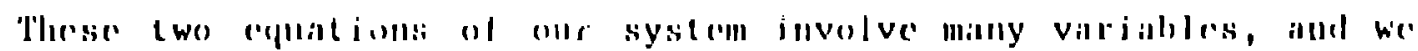

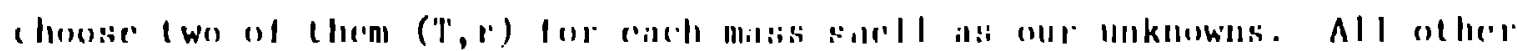
variables, such as the density, lhe pressure, the opacity, the

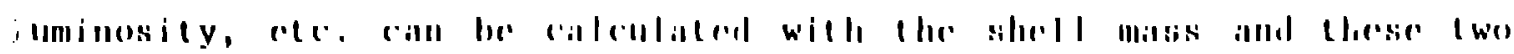

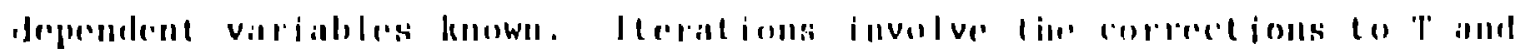

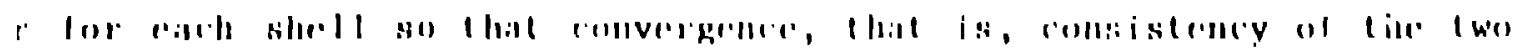

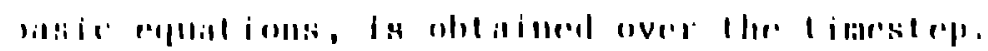

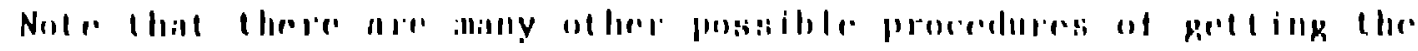

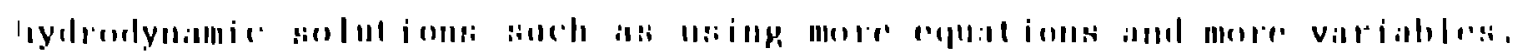

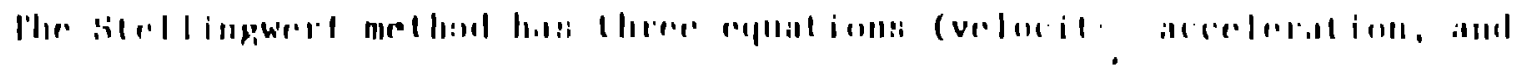


ID IAGRANGIAN HYDRODYNAMICS-TWO VARIABLES ACCELERATION AND NONADIABATIC TERMS

$$
\begin{aligned}
& \ddot{r}_{I}^{n+\frac{l}{2}}==1 / \pi \bar{r}_{I}^{-2}\left(\frac{d P}{d M}\right)_{I}^{n+\frac{1}{2}}-g_{I}^{n+\frac{1}{2}} \\
& \bar{r}_{I}^{2}=1 / 3\left({ }^{n} r_{I}^{2}+{ }^{n_{r_{I}}}{ }^{n+1} r_{I}+{ }^{n+1} r_{I}^{2}\right) \\
& g_{I}=\frac{G M(r)}{n_{r_{I}}{ }^{n+1} r_{I}} \\
& \Delta Q=L_{i n}-{ }_{\text {out }}^{I} \\
& L=4 \pi r_{I}^{2} \frac{\text { ac } v_{I}}{3 K_{I}}\left(\frac{d T^{4}}{d r}\right)
\end{aligned}
$$

Figure 2. The acceleration and nonadiabatic terms are given for the numerical hydrodynamics calculation.

energy) and three unknowns ( $T, r$, and the velocity $u$ ). The KutterSparks system uses five. Thase are the conservation of mass, momentum, energy, the total of the radiative, conductive, and convective luminosjties, and the delinition of velocity. The unknowns here are the $T, r$, V, Llie total luminosity anc ihe velocity. Appenzeller (1970) uses the same equalions but assigrs the unknowns as $T, r$, pressure, the tolal luninosity and the volocity. The backward time differencing (not timestep cencered) that he used was very dissapative, however, even though quite slable. The Castor viriable zone mass (mixed higraligiall and fiulnrian) DYN rode uses an expijicily determ: aed mass together with an implicit solulion for $T, r$, and u using the equations of mass, morentum, and energy.

I nm not sure which systern is the hest. In these completely implicit codes n matrix solution is made for each iteration as we will soon sece, if there nre more epuntions, there nre more rows of the matrix ind more compencints of llac correction vector. This certuinly menes more matrix operations. In our case, it the mumber of equations Is reduced to only two for ciach ache, then the intrix ia more simple.

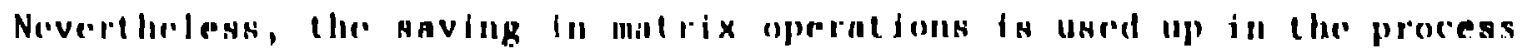

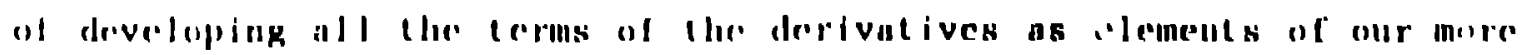
clubognte lwo refunt lons. 
For our two basic equations we need to assign boundar. conditions at the top and bottom of the envelope. The central ball has a fixed radius and a fixed core Juminosity which is the surface luminosity minus any thermonuclear energy sources or neutrino losses in all the envelope zones. At the surface the acceleration of the interface is taken as

$$
\ddot{r}_{I R}=f_{\Lambda} \frac{4 \pi \bar{r}_{I R}^{2}}{M_{I R}}\left(0-P_{I R}\right)=8_{I R}
$$

There the luninosity is calculated by an approximate radiation transport solution for the effective temperature as a function of the temperature at the center of the last zone and the optical depth at that point.

$$
T_{I R}^{4}=3 / 4 T_{e}\left(\tau_{I R} \cdot 2 / 3\right)
$$

With this effective temperature, the luminosity can be calculated from the normal black body luminosity formula

$$
L_{I R}=4 \pi R_{p}^{2} \sigma T_{e}^{4}
$$

As we have indicated before, our implicit method reguires iterations to converge on values of the two dependent variables at the advanced lime, A Nowton-Raphson procedure is rollowed as indicated in ligure 3. The matrix we deal with consists of many derivatives of which there are 2 with respect to $T$ and 3 with respect to $r$ in the momentum cquation, and there are 3 with respect to $T$ and 4 with respect lo $r$ in the encrgy equation. Thus the matrix is 7 diagonal.

Jhe nolalion used in the actual progran is also given in Figure 3. Fach of theac derivatives is calculated each iteration k from sometimes rather complicated formulas. These derivatives are oblained allalylically except for the derivative of the convective luminosity with respect to and $r$. lhese later derivatives are clone numerically by applying an increment of lypically $10^{-7}$ to le and $r$ scparalely and then recalculating the convective luminosity. The difference in the luminosities divided by Lhe difference in the variable ('f or r) gives a partiol derivative which is nceurate conugh to guide the iterations to converg ace.

Ihe Newton-kaphson metherl is applied ly puteino :he negative of the

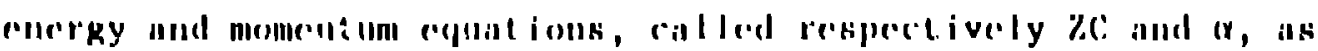




\section{ID LAGRANCIAN HYDRODYNANICS - TWO VARIABLES NEWTON-RAPHSON ITERATIONS}

$$
\begin{gathered}
\alpha_{I}=M_{I}^{k}+\sum_{i=I}^{I+1}\left(\frac{\partial M}{\partial T}\right)_{i}^{k} \delta T_{1}+\sum_{i=I-1}^{I+1}\left(\frac{\partial M}{\partial r}\right)_{i}^{k} \delta r_{1}=0 \\
Z C_{I}=E_{I}^{k}+\sum_{i=I-1}^{I+1}\left(\frac{\partial E}{\partial T}\right)_{i}^{k} \delta T_{i}+\sum_{i=I-2}^{I+1}\left(\frac{\partial E}{\partial r}\right)_{i}^{k} \delta r_{i}=0
\end{gathered}
$$

Band malrix solution for known $M, E$ and derivatives at any iteration $k$ gives the vector $\delta \mathrm{T}_{1}, \delta \mathrm{r}_{1}, \delta \mathrm{T}_{2}, \delta \mathrm{r}_{2}, \ldots \rightarrow 0$.

$$
\begin{array}{ll}
\beta_{I}=\frac{\partial M_{I}}{\partial r_{I}-1} & \mathrm{AC}_{I}=\frac{\partial E_{I}}{\partial T_{I-1}} \\
\delta_{I}=\frac{\partial M_{I}}{\partial r_{I+1}} & B C_{I}=\frac{\partial E_{I}}{\partial T_{I}} \\
\delta_{I}=\frac{\partial M_{I}}{\partial r_{I}} & C C_{I}=\frac{\partial E_{I}}{\partial T_{I}} \\
n_{I}=\frac{\partial M_{I}}{\partial T_{I}} & \mathrm{DC}=\frac{\partial E_{I}}{\partial r_{I}-1} \\
\phi_{I}=\frac{\partial M_{I}}{\partial T_{I}} & \mathrm{EC}=\frac{\partial E_{I}}{\partial r_{I}} \\
& \mathrm{FC}=\frac{\partial E_{I}}{\partial r_{I}} \\
& \mathrm{GC}=\frac{\partial E_{I}}{\partial r_{I}}
\end{array}
$$

Figure 3. Details of the Newton-Riphsson method are given for the illplicit sulat inll. 
components of a right-hand side vector. The linear equations are solved for a correction vector consisting of increments for the $T, r$ pairs. This matrix is portrayed in Figure 4 . Since the matrix elements are changing each iteration, considerable calculation is involved, but usually only 4-5 iterations are needed to get convergence. If the time

- step can be ten or more times that given by the Courant limit, the use of this implicit iteration procedure is warranted.

We need now to discuss the guesses that a:e made for the $T, r$ values for the advanced time $n+1$. In Figure 3 , the momentum and energy equations, that is, $\alpha$ and $\mathrm{ZC}$ for each zone, were time centered between step $n$ and $n+1$. A simple example of this is that in the energy equation the $d E$ is just the internal energy at time $n+1$ minus its value at time $n$. Consider now the momentum and energy equations at time $n+\frac{1}{2}$, in order to make our extrapolation or guesses for time $n+1$. A Taylor expansion of the two equations would result in almost exactly the two expressions given at the tcp of Figure 3, and they both equal zero because these two equations are here assumed to be zero at the mean time. The Taylor expansion, however, involves only quantities at time $n$ and none at time $n+1$. These equations at time $n$ and their various derivatives are somewhat different than they were in the implicit method itcrations which involve time contered quantities. If the extrapolation is made to the mean time, the derivative terms should be multiplied by $\frac{1}{2}$. Solution for the correction vector, as described before, now allows them to be applied to give a very good approximation to the configuration at time $n+1$, and a good stirling point for the jterations. We call this Taylor expansion the explicit $P$ step and the iterations as the $F$ step of the time step.

In DYNSTAR, all the lime centered quantities arr exactly half of their time $n$ value and half of the time $n+l$ value except for the luminosities. following stellingwerf (1975), we give the $n+1$ Juminositics a weight of $2 / 3$ and those at $l$ ime $n$ only $1 / 3$. Presumably this makes the integrations more stable without much loss in accuracy. With our centering at exa ly at the time midpoint, the procedure is margillialy stable, and somelimes we hilve convergence trouble for large timest eps. 


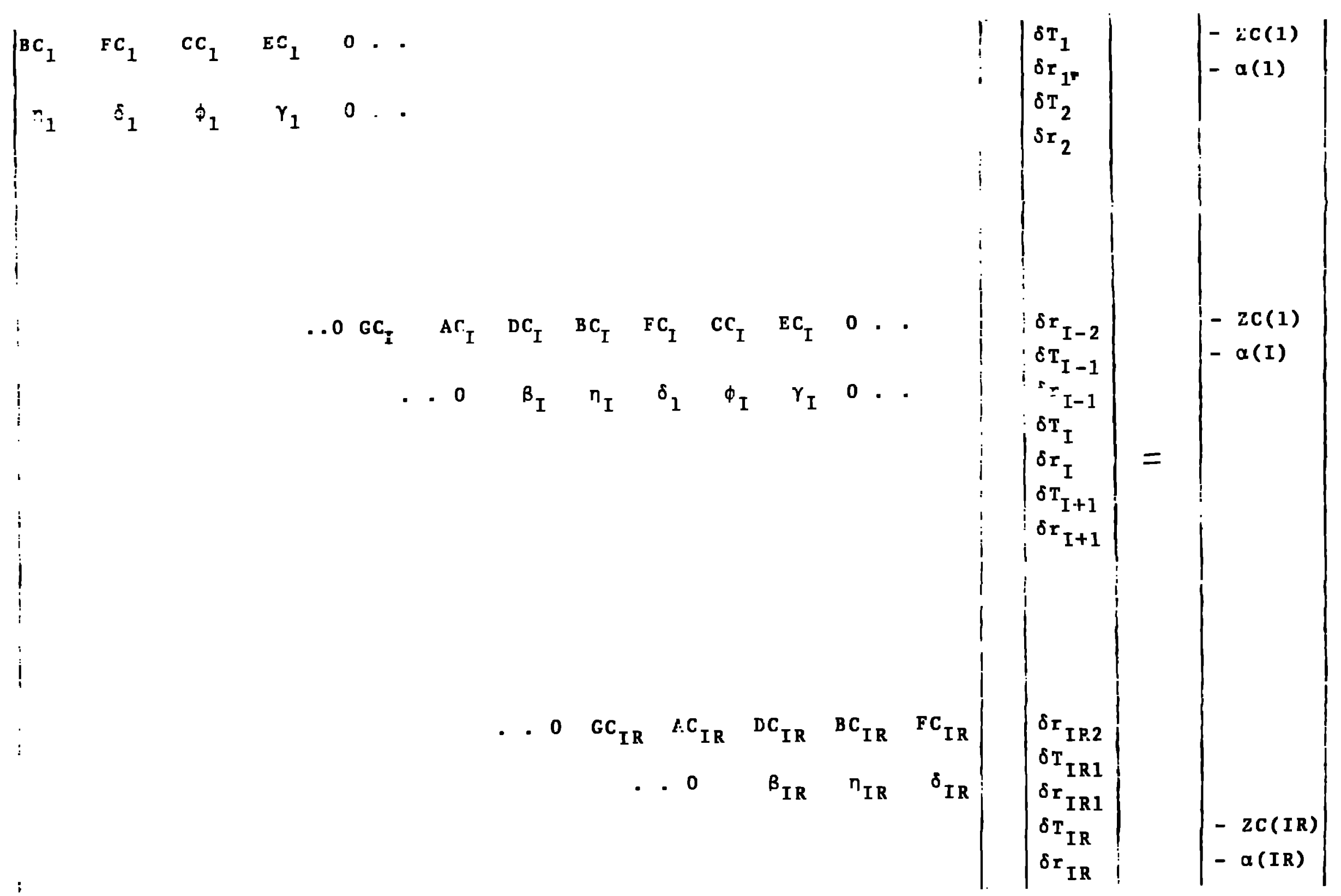

Figure 4. The structure of the 7 diagonal matrix for the solution for the correction vector is displayed using the quantities defined in the previcus figure. 
A very importart thing to do when using the Newton-Raphson method is to be sure that the largest increment in the $T$, $r$ correction vector is not so large that the solution iteratius are upset ond nonconvergent. In the normal case, the maximum, wherever it is in the zones, can be as large as $30 \%$, but sometimes a much smaller correction needs to be -applied so that the equations do not stray out of their radius of convergence.

Time dependent convection is necessary to give realistic hydrodynamic solutions in most cases. Figure 5 presents the nixing length aquations with a modification for the convective velocity variation. As used today, the increase of the convective velocity each time step is $0 . i$ or less times the velocity of an element $(\Delta v)$ after it has travelled a mixing length. The decrease is given by the fomula for the lag, and if the gradient becomes subadiabatic, the drag is multiplied by 1000 . These parameters do not matter much for upper main sequence stars, because the surface convection zone is very weak and the core convection timescale is perhaps 100 times the pulsation period.

All of the usual hydrodynamic codes, for pulsation or not, need an artificial viscosity to smooth out strong disturbances such as shock waves. The use of the viscosity is simple. The pressure which consists of gas and radiation terms as well as perhaps the convective turbulent pressure term, is further increased by the turbulent eddy and artificial viscosity terms. This pressure, used both in the momentum and energy equations, is given as

$$
\begin{aligned}
& q_{I}=q_{I}+q t_{I} \\
& q a_{I}=\ddot{v}_{1}^{F A}\left[\dot{r}_{I}^{n+1}-\left(r_{I-1} / r_{I}\right)^{2} \dot{r}_{I-1}+\theta v S_{I}\right]^{2} \\
& \text { if }[\quad l \text { term } 0 \\
& q a_{I}=0 \text { otherwise } \\
& q l_{I}=\underset{v}{F} v_{c} \wedge_{r} d \dot{r}
\end{aligned}
$$

$v_{c}$ and $\wedge_{e}=$ (rlly) Irom $t$ ime dependent mixing length theory 
TIME DEPENDENT CONVELiION

$$
\begin{aligned}
& \text { If } v_{c} \geq v_{c}^{n} \quad v_{c}^{n+1}=v_{c}^{n}+\Delta v \\
& \Delta v=\frac{\Delta \rho}{\rho} \& \frac{2 \Lambda}{r_{i+1}-r_{i-1}} \Delta t \quad A F \geq 0 \\
& \frac{\Delta \rho}{\rho}=Q \frac{\Delta T}{T} \\
& \text { if } v_{c}<v_{c}^{n} \\
& v_{c}^{n+1}=v_{c}^{n}+\tau\left(v_{c}-v_{c}^{n}\right) \geq 0 \\
& \tau=\frac{v_{c}^{n}+v_{c}^{n+1}}{2} \frac{\Delta t}{\Lambda} L F \\
& L_{c} \sim v_{c}^{3} \\
& L F=1 \\
& \text { IF } v_{c}<0 \quad \frac{\Delta \rho}{\rho}<0 \text { use } \Delta v<0 \\
& \text { Wilh } \quad A F=1000
\end{aligned}
$$

Figure 5. The equalions for the time dependent convection are based on a la of the convective element velocity in mixing length theory.

We somelimes use a linear artificial viscosity where the square brackel term is not squared, the threshold $\theta$ is set to 0 , and a factor of the sound velocily is added.

Material propertieg are nceded lo carry oul ralculations. We have alreary discussed the opacitics, and the equation of state needed to calculate them is also necded to calculate the pressure and energy fo. the mass shelle in the hydrodynamirs integrations. Bnth tabular and analytle formulas are usid, the laller when it scems very necessary to oprerate with smoolh opacily and rquation of stale derivatives as for 
convection or just for ease of the jteration convergence. The standard thermonuclear reaction rates of Fowler, Caughlan, and Zimmerman (1975) and screening factors discussed by Reeves (1965) are used for any energy source present.

Opacjties vary rapidly with temperature and they are known only at

- the center of the mass shells in our hydrodynamic calculations. Yet, the radiative luminosity passing :rom shell tc shell is space centered at the shell interfaces. Simple averaging of the opacities in adjacent zones is not adaquate especially if the number of zones is limited $L_{0}$ typically 50-100. Christy (1967) and Stellingwerf (1975) have proposcd averaging procedures. hilhout giving the procedure here, we merely say that wr use the Stellingwerf method.

Selection of the time step is crucjal for the calculations. If the step is too small, results will be slow in colling. If the time step is too large, the ilerations will nol converge. We hive adopted a lime step ielection procedure that retains l.he previous time step if the number of itcations for the last time step was reasollabe, say six to ten. For a larger number of iteralions, the problem is having trouble and the linc slep is coll by $15 \%$. If convergence is very casy (in less than six iteralions), multiplication of the timestep by 1.35 is made for llie next one.

Ihe typial wity a pulsition solution is started is to lase the

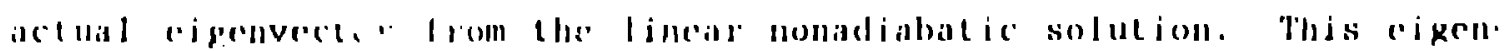
vector is rescouled from its asual formalizalion hilh a surlace ampliturle

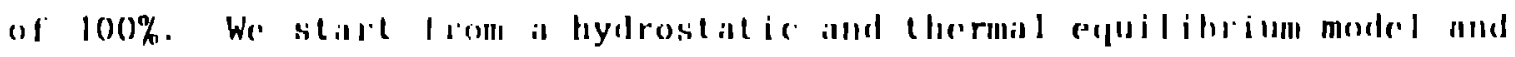
apply the radius egenvector as the outhild velocity structure. To get the 'I, I contigulat ion that ohe ails al this milpulsilt ion position we

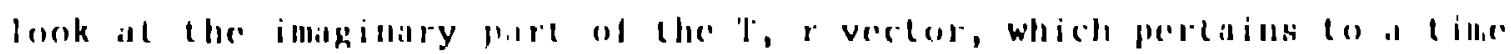

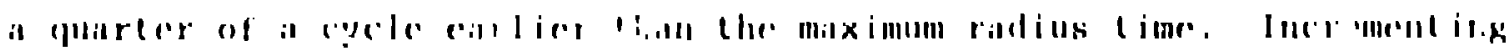

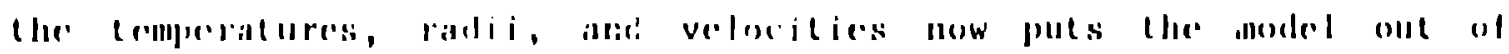

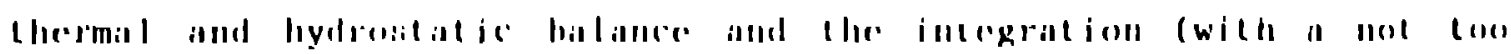
l.upg timesterl) begitus.

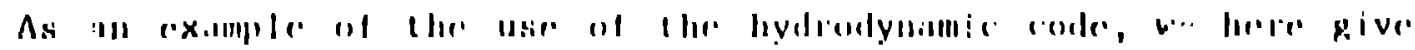

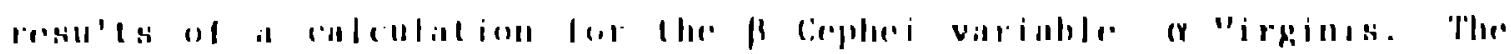

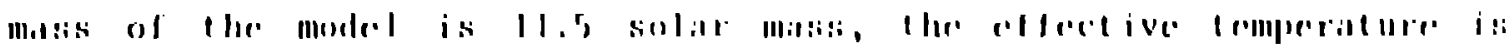

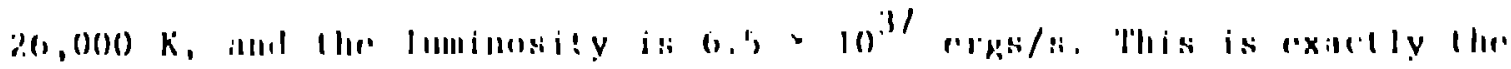

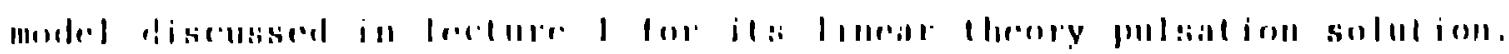


This linear solution has been scaled here to a photospheric pedt to peak radial velocity of $24 \mathrm{~km} / \mathrm{s}$ to allow for the limb darkening which affects the $17 \mathrm{~km} / \mathrm{s}$ observed amplitude.

Figure 6 shows the radial velocity variations for about 10 periods. All cycles do not repeat exactiy in this calculation. Figure 7 is the -radius variation from the hydrodynamic integration, and a peak-to-peak variation of about $1 \%$ is seen. Since all the interior variations are even smaller, and the decay e-folding rate for this fundamental mode is only about one part in 10,000, it is reasonable to expect that this model is behaving very closely to the result from linear adiabalic theory.

Bolometric magnitude variations are displaycd in Figure 8 . Here the amplitude peak-to-peak is 0.19 magnilude which corresponds to a much smaller amplitude in the visual band. The surface effective temperature variations of $650 \mathrm{~K}$ above ang below the mean of $26,000 \mathrm{~K}$, given in Figure 9, produce a varyjing lolometric correction of close to 0.11 magnitude (Code et al.1975) with the maximum correction occuring at the hotlest phase which is also the most lumininus phase at minimum radius. These corrections are -2.63 at $26,650 \mathrm{~K}$ and -2.52 at $25,350 \mathrm{~K}$. subtaction of this correction lifference then produces somenhat more than the actual tew hundredths of a magnitude chserved variation in the V filerer.

The kinetic energy of the motions vilies as shown in figure 10 . Twi.d rach rycle, at maximum infall and moximum expansion velucity, the kinetis colergy reaches a maximum. For other stars that are not so adialiatic, there ine platse differences between the maximum velocity at different levels in the stir, and the peaks and valleys of this kinctic chergy varialion ire nol so separieged as in our ciase where there is a range of three or more powers of tell.

We believe that the supergiallt pulsations ale all nonralial,

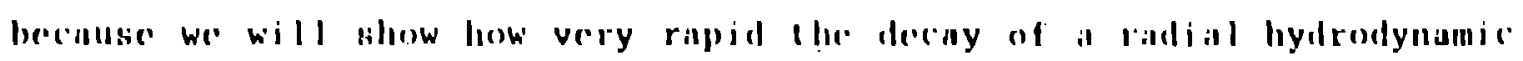
asturbilace is in cur linil lecelure. 


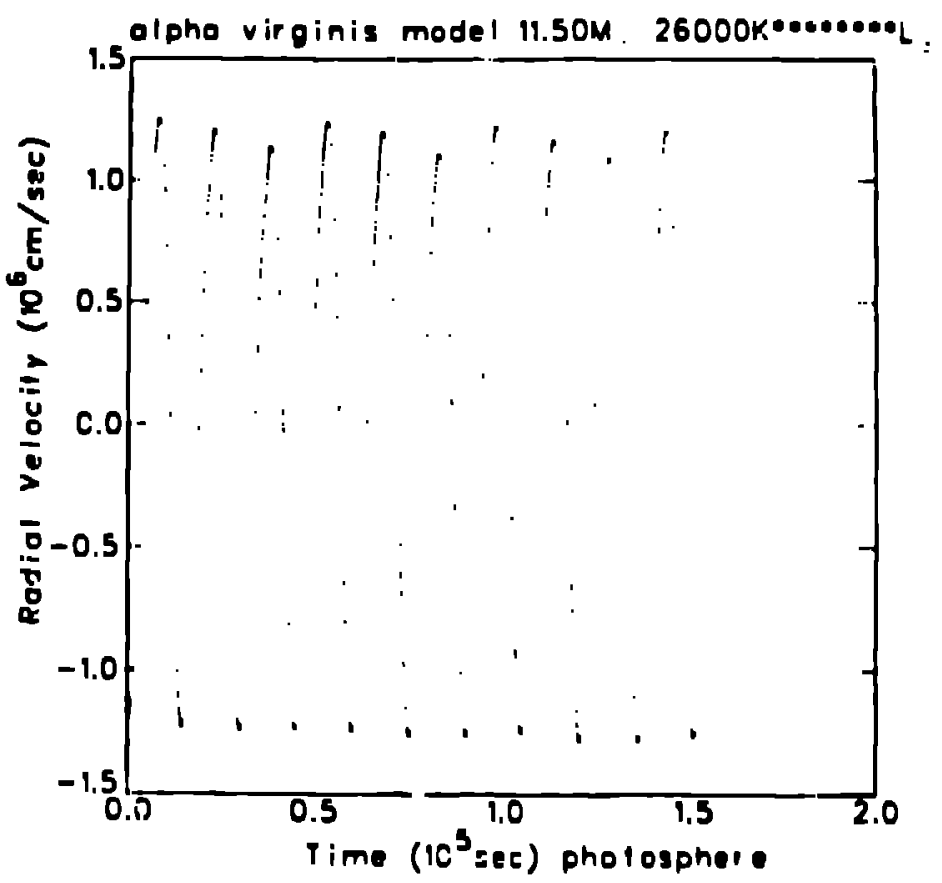

Figure b. Ihe radial velocity histery is showill for the hydrodynamic study of all er l'ir medel.

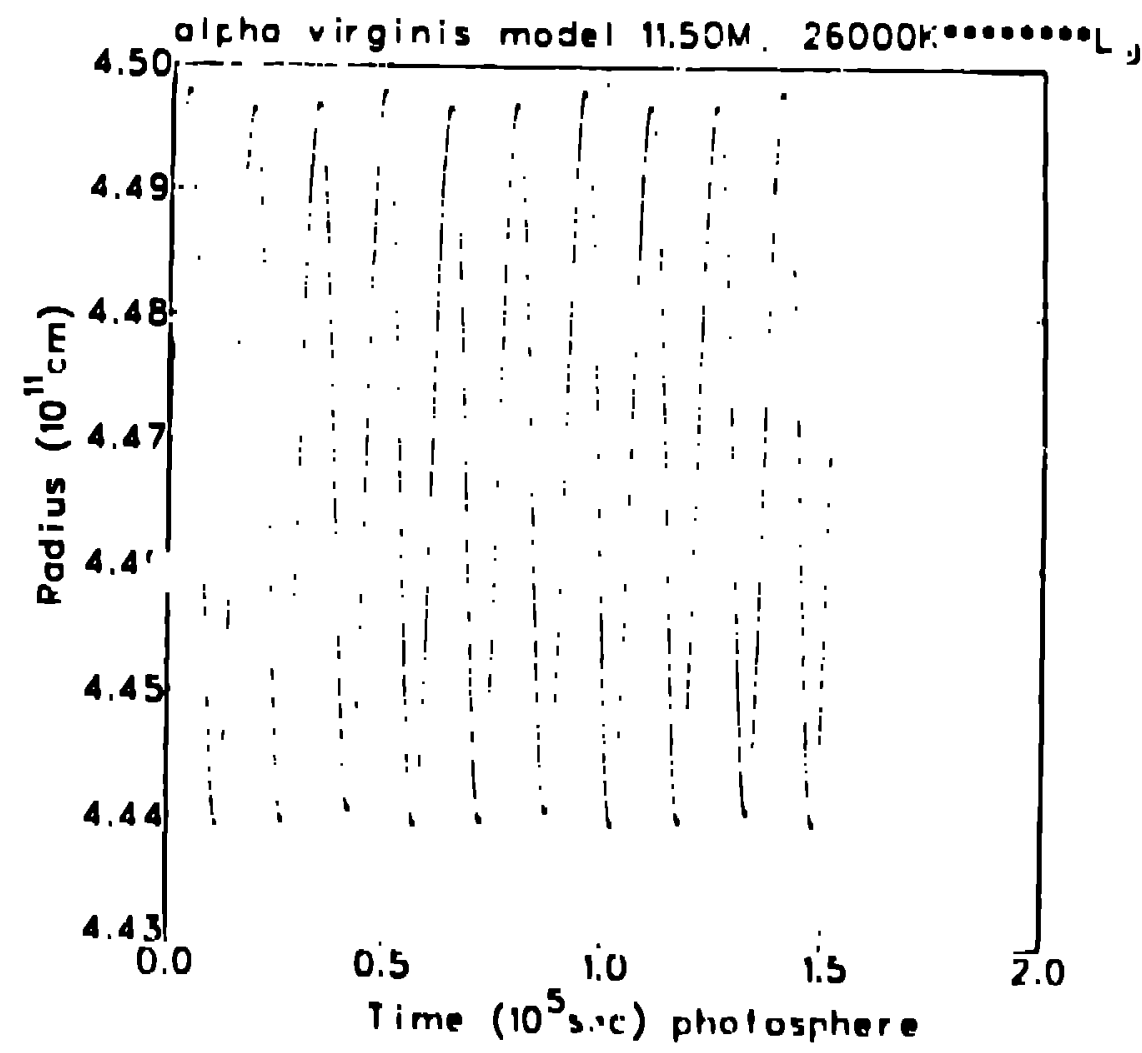

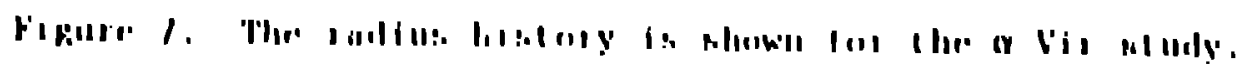




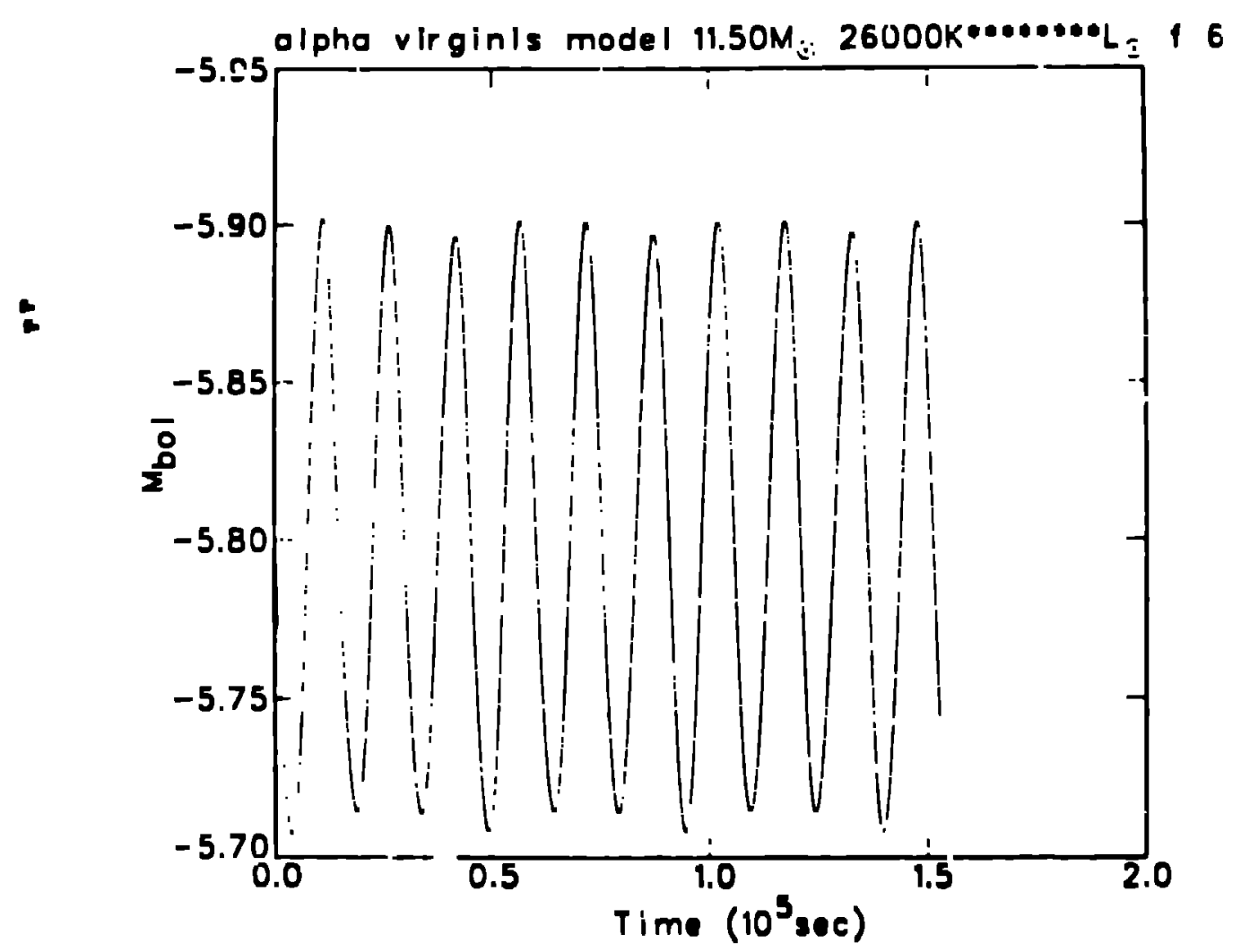

Figure 8. Thc luninosity history is given for th a Vir study.

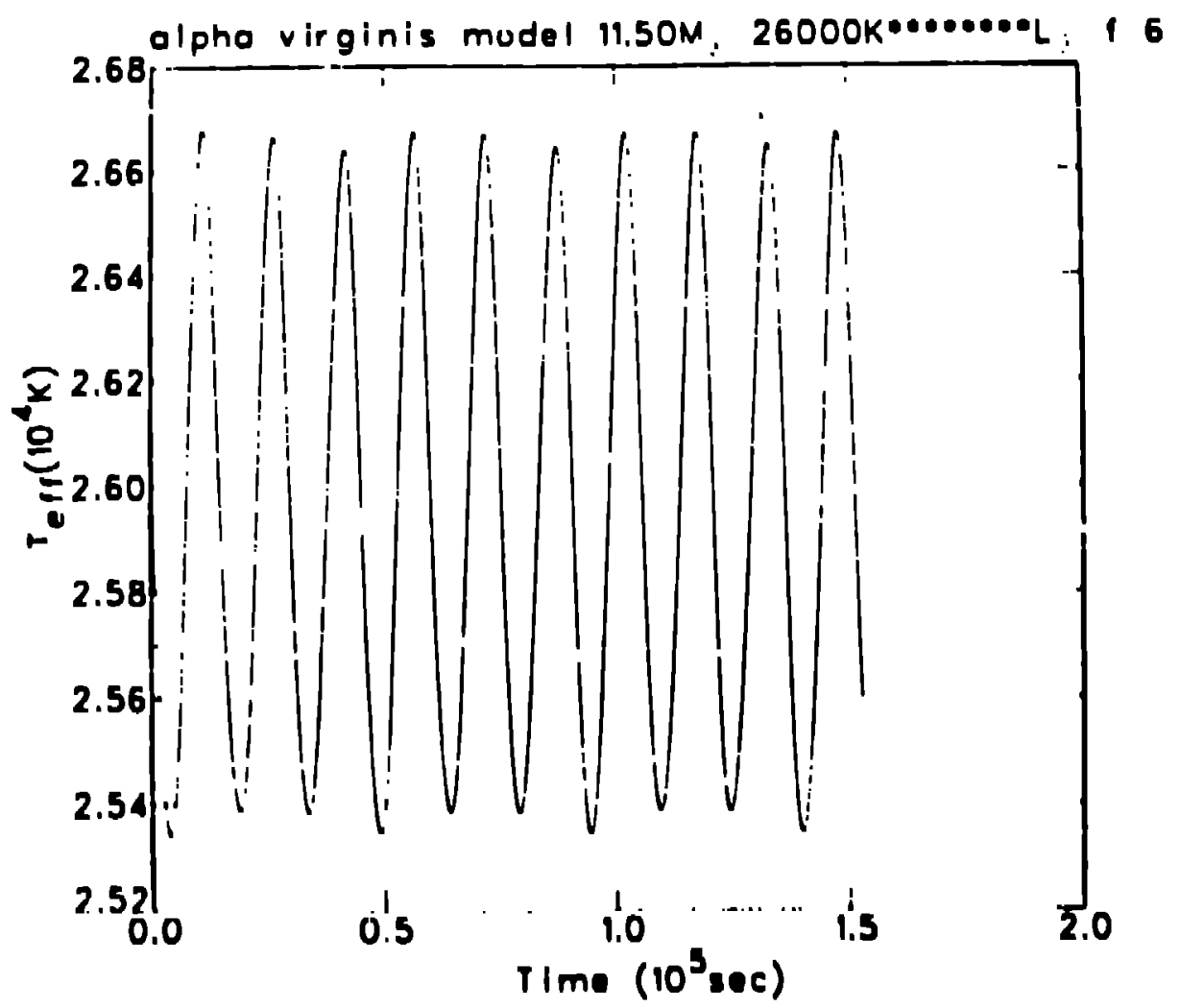

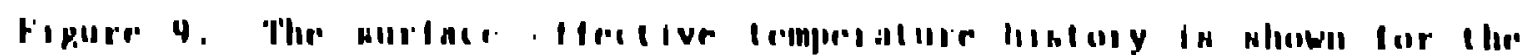
II VIr: $: 1$, ily. 


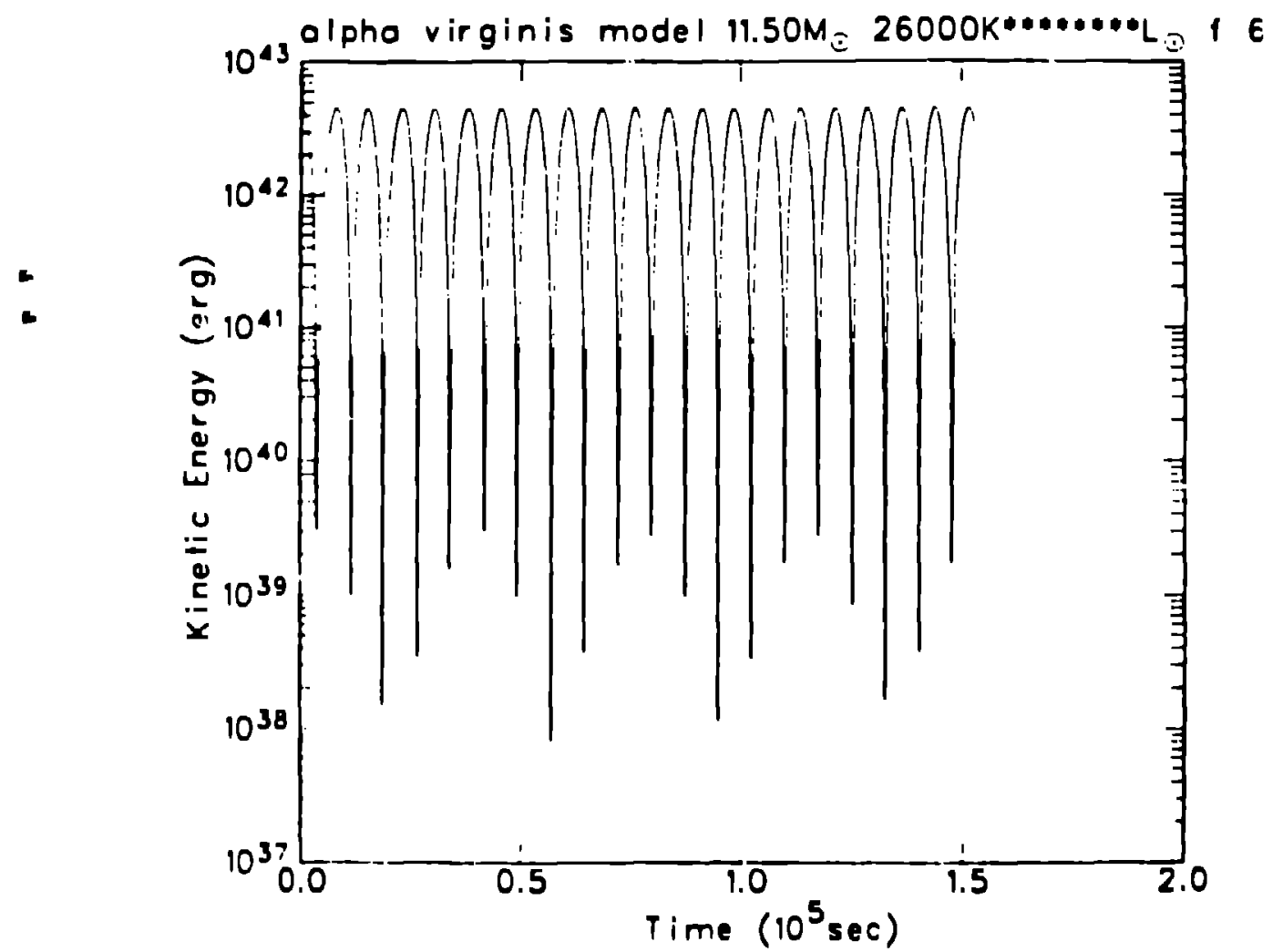

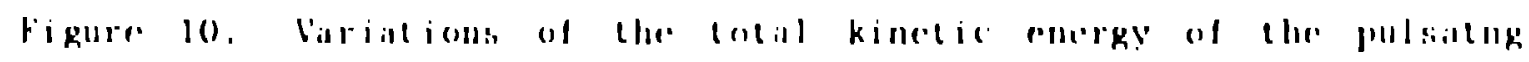

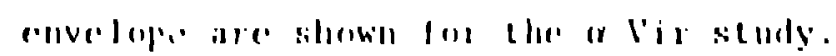




\section{RFFERENCES}

Appenzeller, I. 1970, Astron. Astrophys. 5, 355.

Baker, N.H. and von Sengbusch, K. 1969, Mitt. der Astron. Gesellschalt, 27,162 .

- Bendt, J.F. and Davis, C.G. 1971, Ap. J. 169, 333.

Lastor, J.J. 1971, Ap. J. 166, 109.

Castor, J.1., Davis, C.G., and Davison, D.K. 1977, Los Alamos Repl $1 . A-6664$.

Christy, R.F. 1964, Rev. Mod. Phys. 36, 555.

Christy, R.F. 1967, Method Comp. Phy.. 7 (Acadelli: Press, New York) 1!1.

Code, A.D., Divis, J., Bless, K. C., and Brown, R. H. 1976, A\}. J. 203, 417.

Cox, A.N., Hrownle(e, R.R., alld Fiters, D.l). 1966, Ap. J. 144, 1024.

Fowler, W.A., Calughlin, G.W., ind Zimmermall, H.A. 1975, Ann. Rev. Astron. Astrophys. 13,69 .

Fialey, (i.s. 1068, Ast. Spince Sci. 2, 's,

Knrp, A.ll, 1975, Ap. J, 199, 144.

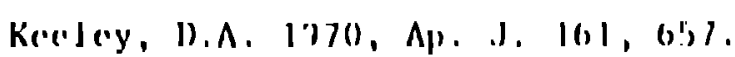

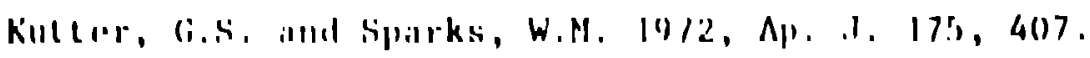

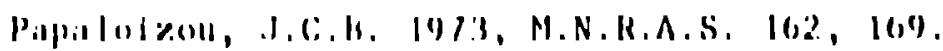

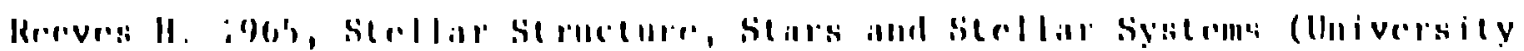
of (hillyolloses) pli3.

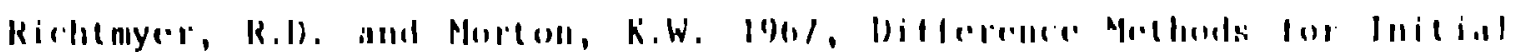
Vil lue l'ublimes (Interescience, Neb! York).

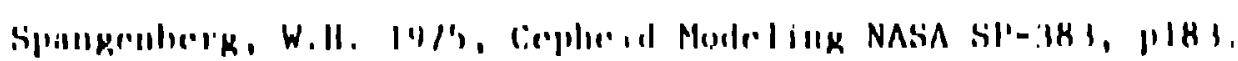

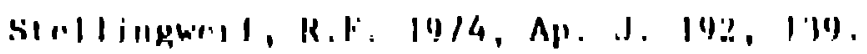

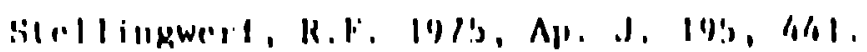

Stolite, R.S. (1)(1), M.N.R.A.S. R4, hol,

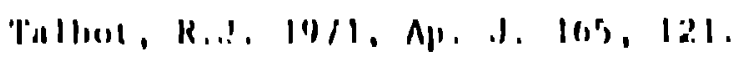

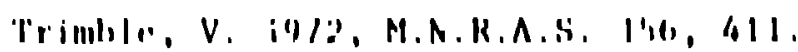

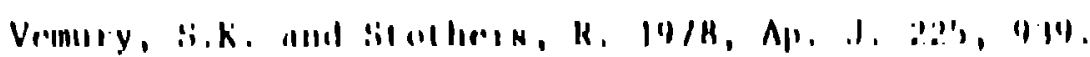

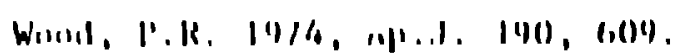

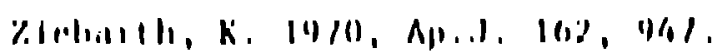

